\title{
Abdominal aortic aneurysm in giant cell arteritis
}

\author{
Hyunwook Kwon, Youngjin Han, Da Hye Son', Yong-Pil Cho, Tae-Won Kwon \\ Division of Vascular Surgery, Department of Surgery and ' Department of Pathology, Asan Medical Center, University of Ulsan \\ College of Medicine, Seoul, Korea
}

Aortic complications of giant cell arteritis are a rare cause of abdominal aortic aneurysm. Here, we describe a case of a ruptured aortic aneurysm in a patient with giant call arteritis (GCA) who was preoperatively suspected of having an infectious aortic aneurysm. Intraoperative inspection revealed infectious granulation tissue on the anterior wall of the abdominal aorta. GCA was finally confirmed by pathological diagnosis. Our findings suggest that the surgical and postoperative treatment of nonatheromatous aortic aneurysm should be based on accurate diagnosis.

[Ann Surg Treat Res 2015;89(4):224-227]

Key Words: Giant cell arteritis, Aortic aneurysm, Aortitis

\section{INTRODUCTION}

Giant cell arteritis (GCA) is a systemic vasculitis that usually affects people older than 50 years and predominantly those with large- and medium-sized arteries [1]. Although branches of the external carotid artery are preferentially involved, GCA can occur in extratemporal large vessels such as arteries of the upper and lower extremities as well as the aorta. GCA is rare, but is the most common cause of nonatheromatous aortic aneurysm. Aortic involvement in GCA is generally recognized in the acute phase because most patients are asymptomatic [2]. Because most patients with infected aortic aneurysms have abdominal and/or back pain, the differential diagnosis between infected aneurysm and symptomatic aortic involvement in GCA is difficult. This report describes a case of a ruptured abdominal aortic aneurysm (AAA) that was preoperatively suspected of being an infectious aortic aneurysm and finally confirmed as GCA by histopathologic diagnosis.

\section{CASE REPORT}

A 57-year-old man was referred for an infrarenal AAA with signs of impending rupture. His past medical history showed no cardiovascular risk factors except for arterial hypertension and a smoking history of 30-pack years. The patient reported lower back pain persisting for 3 weeks and aggravation of abdominal pain over the course of 1 week. Initial blood pressure was $135 / 88 \mathrm{mmHg}$ and heart rate 98 beats/min. The patient had no fever. A physical examination revealed mild tenderness around the umbilicus; however, no pulsatile mass was identified. Laboratory tests showed an elevated CRP level of $7.99 \mathrm{mg} / \mathrm{dL}$ and an erythrocyte sedimentation rate of $51 \mathrm{~mm} /$ $\mathrm{hr}$; all other blood test results including white blood cell count, hemoglobin, chemical, and electrolyte levels were normal. Preoperative $\mathrm{CT}$ scan showed an AAA measuring $8 \mathrm{~cm}$ in extent from $5-\mathrm{cm}$ distal to the renal artery to the aortic bifurcation with a maximal diameter of $7.6 \mathrm{~cm}$. The aneurysm sac was filled with eccentric high-attenuated mural thrombus suspected to be an impending rupture (Fig. 1).

Intraoperative inspection revealed a ruptured AAA with infectious granulation tissue on the anterior wall with mural thrombosis (Fig. 2). We performed a complete excision of the aneurysm and Y-graft interposition with GORE-TEX graft (W. L. Gore and Associates, Flagstaff, AZ, USA). The graft was wrapped
Received April 1, 2015, Revised May 19, 2015, Accepted June 5, 2015

\section{Corresponding Author: Tae-Won Kwon}

Department of Surgery, Asan Medical Center, University of Ulsan College of Medicine, 88 Olympic-ro 43-gil, Songpa-gu, Seoul 05505, Korea

Tel: +82-2-3010-3492, Fax: +82-2-3010-6701

E-mail: twkwon2@amc.seoul.kr
Copyright (c) 2015, the Korean Surgical Society

(c) Annals of Surgical Treatment and Research is an Open Access Journal. All articles are distributed under the terms of the Creative Commons Attribution NonCommercial License (http://creativecommons.org/licenses/by-nc/4.0/) which permits unrestricted non-commercial use, distribution, and reproduction in any medium, provided the original work is properly cited. 

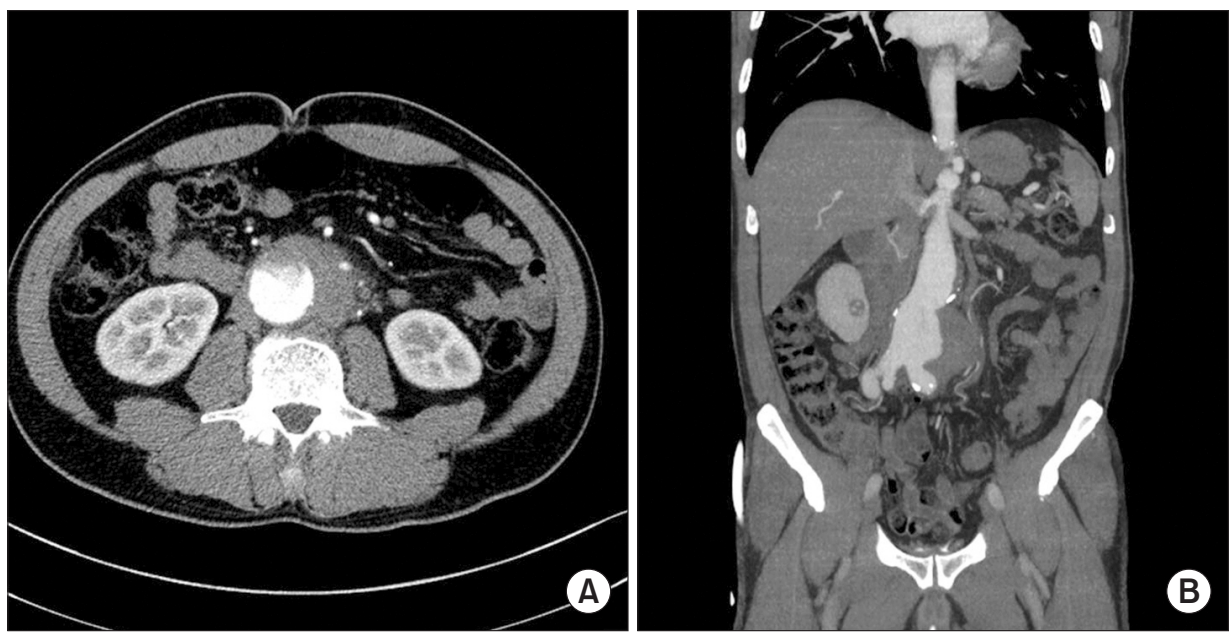

Fig. 1. Preoperative contrast enhanced computed tomography: (A) axial and (B) coronal views showing an infrarenal abdominal aortic aneurysm with evidence of an impending rupture and eccentric high attenuated mural thrombus.
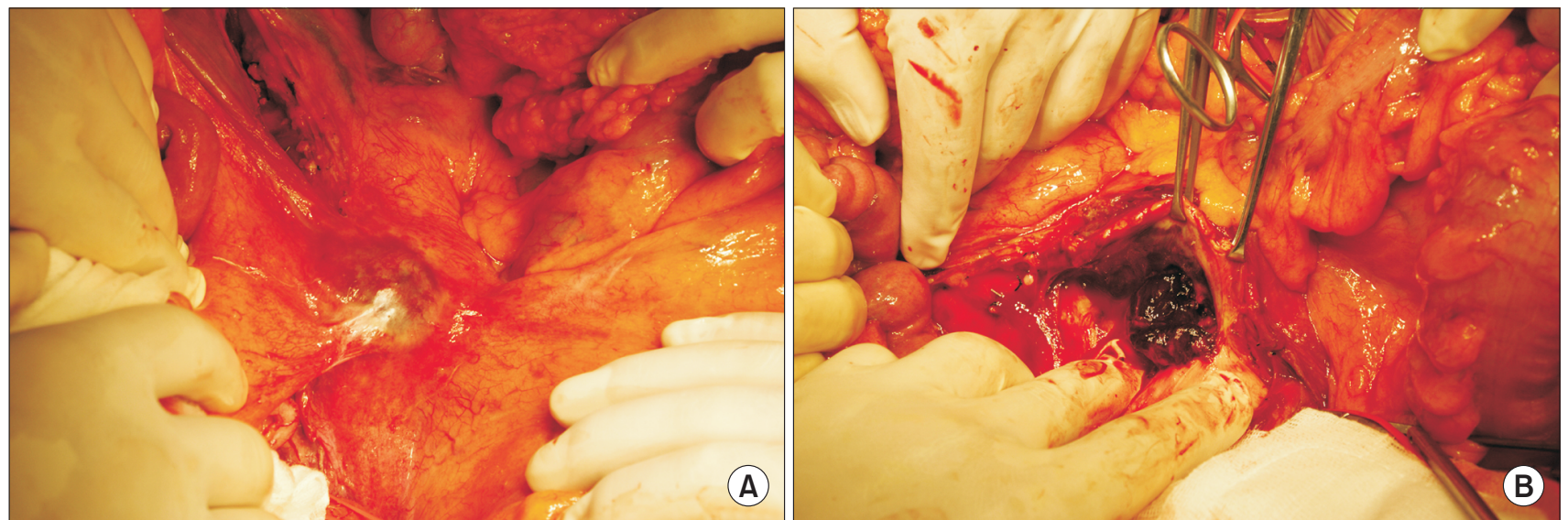

Fig. 2. (A) Intraoperative findings revealing a ruptured aortic aneurysm with infectious granulation tissue on the anterior wall. (B) Fresh thrombosis could be seen during aneurismal sac dissection.
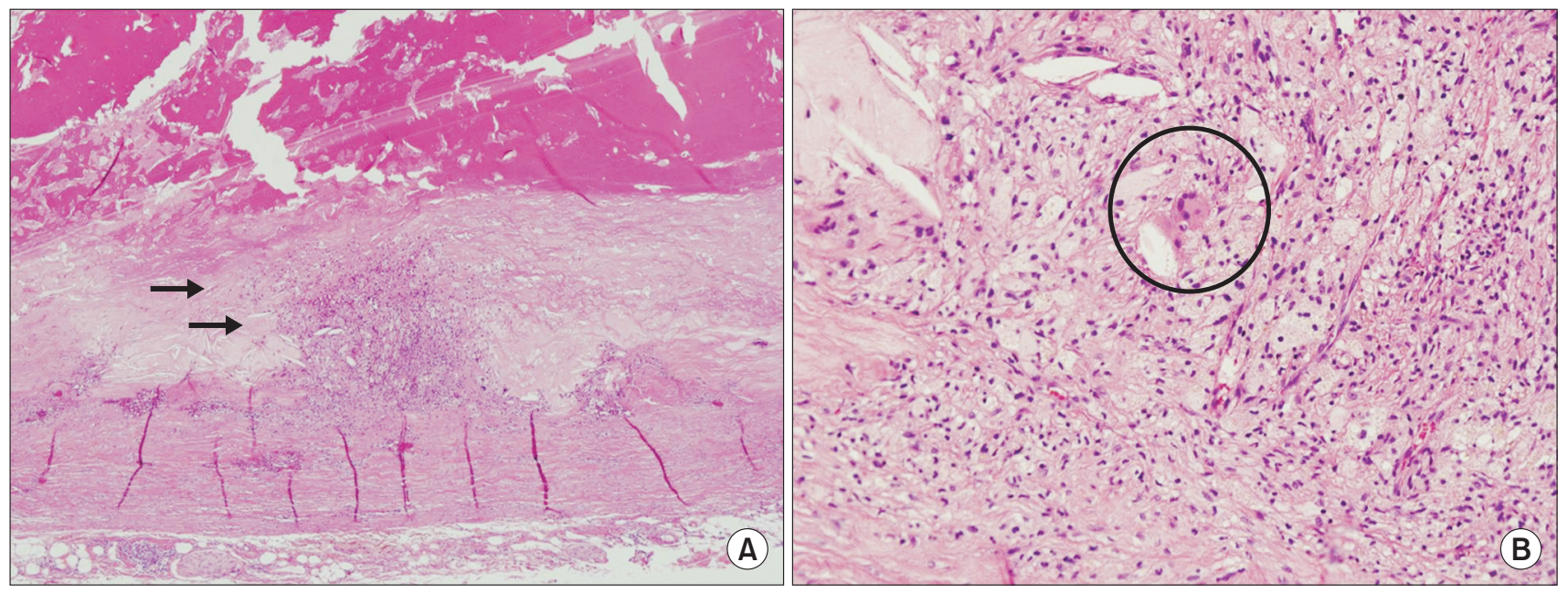

Fig. 3. Histopathologic analysis of the resected aneurismal sac (H\&E staining). Arrows indicate intimal thickening and thrombosis leading to luminal narrowing, and inflammatory cell infiltration (A), consisting of mixed chronic inflammatory cells and occasional giant cells (circle) (B). 
with an omental flap. No microorganisms were founded on blood or tissue culture. Laboratory results at the time of discharge revealed a normal CRP level, but ESR level remained high at $50 \mathrm{~mm} / \mathrm{hr}$. Histopathologic analysis of the resected aneurismal sac showed complete loss of the media focally in the elastic staining and extensive perivascular fibrosis consisting of chronic inflammatory cells and occasional giant cells, suggesting giant cell aortitis (Fig. 3). The patient was discharged 9 days after surgery with unremarkable postoperative course. At postoperative 15 months, CT scan showed patent graft and distal run-off and no abnormal postoperative finding. The patient has developed no other symptoms related to GCA.

\section{DISCUSSION}

The diagnosis of GCA is challenging and usually based on clinical manifestation and biopsy. Histologic features showing inflammatory infiltrates in the arterial wall often with giant cells mostly at the border between the intima and the media are the most conclusive evidence for a diagnosis of GCA [3]. The temporal artery is the most common biopsy site because it is easy to access and is a frequently involved site. Although biopsy is a confirmative method, $19 \%$ of cases show false-negative on biopsy probably due to missed lesions [4]. In patients who only show symptoms of polymyalgia rheumatic (PMR), only a small portion (4.4\%) are positive on temporal artery biopsy [5]. In the present case, the patient revealed no previous symptoms indicating GCA except lower back pain persisting for 3 weeks ago prior to presentation. However, histologic findings from a specimen obtained during surgery indicated necrotizing aortitis with giant cells.

Clinical manifestation of disease should be considered alongside biopsy results. Temporal arteritis with headache, tenderness, jaw claudication, and ophthalmic symptoms is the most typical presentation of GCA. Systemic inflammatory signs, PMR, and laboratory results such as high ESR, high CRP, anemia, and thrombocytosis are also indicative of GCA. The 1990 American College of Rheumatology GCA classification criteria suggests five parameters: (1) age at onset $\geq 50$ years, (2) new onset localized headache, (3) temporal artery tenderness or decreased pulsation, (4) ESR $>50 \mathrm{~mm} / \mathrm{hr}$, and (5) abnormal artery biopsy. Our patient presented three of these five criteria, namely age, ESR, and biopsy, with a sensitivity of $93.5 \%$ and a specificity of $91.2 \%$ [6].

Aortic involvement is generally asymptomatic and concurrently identified at the time of GCA diagnosis, while aortic involvement after diagnosis of GCA is rare $(14.6 \%$ of patients). In symptomatic aortitis, patients can manifest back pain, abdominal pain, and dyspnea [2]. The incidence of aortic aneurysm and/or dissection in patients with GCA is reported to range between $9.5 \%$ and $17.9 \%$ [7]. Hypertension,
PMR, and an aortic insufficiency murmur have been suggested as possible predictors of aortic aneurismal disease in GCA patients [7]. Marie et al. [2] reported that $72.7 \%$ of cases of nonatherosclerotic aortic involvement resulted from GCA while $16.7 \%$ of nonatheromatous aortic lesions were associated with infection. Almost all patients with infected aortic aneurysms present with abdominal pain and fever. In addition to clinical manifestations, the presence of leukocytosis, results of an intraoperative culture, and conditions related to aortic infection should be considered for the diagnosis of infected aortic aneurysm [8]. Since our patient complained of abdominal and back pain and had elevated preoperative CRP and ESR levels, we initially suspected an infected aneurysm.

Corticosteroid can be used to reverse the symptoms of GCA guided by clinical activity and levels of inflammatory parameters such as ESR and CRP. Garcia-Martinez et al. [9] treated 54 biopsy-confirmed GCA patients using a steroid protocol consisting of $1-\mathrm{mg} / \mathrm{kg} /$ day prednisolone for 1 month and tapering off at $10 \mathrm{mg} / \mathrm{wk}$. Among them, 12 patients (22.2\%) had aortic aneurysm/dilatation, five of who required surgical repair. Marie et al. [2] reported that four of seven patients who developed aortic involvement after the onset of GCA were receiving prednisolone. Although corticosteroid can elicit acute symptoms, it may be inadequate to prevent aortic involvement [1].

To date, no confirmative strategies exist to screen and follow up GCA patients for the early detection of aortic aneurysm. Several studies suggest annual chest radiography, abdominal ultrasound with careful medical interview, and physical examination. Laboratory testing such as for ESR and proinflammatory cytokines may also provide useful information on the patient's inflammatory status [10]. CT-scan can help in establishing diagnosis and for follow-up of aortic involvement in patients with GCA. Although positron emission tomography with fluorodeoxyglucose or MRI is useful for early diagnosis, routine monitoring with PET or MRI has provided no benefit in terms of disease management. Therefore, CT or MRI may be a good choice for screening of high-risk patients [10].

In conclusion, our present case presents a rare example of AAA associated with GCA. The surgical and postoperative treatment of nonatheromatous aortic aneurysm should be based on accurate diagnosis. Open surgical procedures and adequate antibiotic treatment according to results from cultures of the aortic wall or blood is the standard treatment for infected aneurysms [8]. However, if GCA is incidentally identified after aortic surgery, the remaining large- and medium-sized arteries should be monitored and corticosteroid can be used to resolve acute symptoms of GCA. Surgeons who encounter nonatheromatous aortic aneurysms should assure a final diagnosis and plan appropriate management. 


\section{CONFLICTS OF INTEREST}

No potential conflict of interest relevant to this article was

reported.

\section{REFERENCES}

1. Borchers AT, Gershwin ME. Giant cell arteritis: a review of classification, pathophysiology, geoepidemiology and treatment. Autoimmun Rev 2012;11:A544-54.

2. Marie I, Proux A, Duhaut P, Primard E, Lahaxe L, Girszyn N, et al. Long-term follow-up of aortic involvement in giant cell arteritis: a series of 48 patients. Medicine (Baltimore) 2009;88:182-92.

3. Lie JT. Illustrated histopathologic classification criteria for selected vasculitis syndromes. American College of Rheumatology Subcommittee on Classification of Vasculitis. Arthritis Rheum 1990;33:1074-87.

4. Breuer GS, Nesher R, Nesher G. Negative temporal artery biopsies: eventual diagnoses and features of patients with biopsy- negative giant cell arteritis compared to patients without arteritis. Clin Exp Rheumatol 2008;26:1103-6.

5. Myklebust G, Gran JT. A prospective study of 287 patients with polymyalgia rheumatica and temporal arteritis: clinical and laboratory manifestations at onset of disease and at the time of diagnosis. Br J Rheumatol 1996;35:1161-8.

6. Hunder GG, Bloch DA, Michel BA, Stevens MB, Arend WP, Calabrese LH, et al. The American College of Rheumatology 1990 criteria for the classification of giant cell arteritis. Arthritis Rheum 1990;33:1122-8.

7. Gonzalez-Gay MA, Garcia-Porrua C, Pineiro A, Pego-Reigosa R, Llorca J, Hunder GG. Aortic aneurysm and dissection in patients with biopsy-proven giant cell arteritis from northwestern Spain: a population-based study. Medicine (Baltimore) 2004;83:335-41.

8. Fillmore AJ, Valentine RJ. Surgical mortality in patients with infected aortic aneurysms. J Am Coll Surg 2003;196:435-41.

9. Garcia-Martinez A, Hernandez-Rodriguez J, Arguis P, Paredes P, Segarra M, Lozano E, et al. Development of aortic aneurysm/ dilatation during the followup of patients with giant cell arteritis: a cross-sectional screening of fifty-four prospectively followed patients. Arthritis Rheum 2008; 59:422-30.

10. Bongartz T, Matteson EL. Large-vessel involvement in giant cell arteritis. Curr Opin Rheumatol 2006;18:10-7. 